\title{
Approaches to assessing the characteristics of a vehicle body based on a virtual test bench
}

\author{
Elena Zhilenkova ${ }^{1, *}$, Pavel Cvetkov ${ }^{1}$, and Ignat Epifantsev ${ }^{2}$ \\ ${ }^{1}$ Peter the Great St. Petersburg Polytechnic University, 195251, St. Petersburg, Russia \\ ${ }^{2}$ Saint Petersburg State Marine Technical University, 190121, St. Petersburg, Russia
}

\begin{abstract}
The paper deals with the problem of assessing the characteristics of a vehicle body on the example of a car body. Such systems allow not only to reduce the cost of design, but also to obtain a number of important estimates that are not available at the stage of full-scale tests. An assessment system based on a virtual platform and a digital twin is considered. It is shown that the acceptable accuracy of the estimates is provided by the sufficient accuracy of the mathematical model. Considerations on approaches to designing the structure of such software systems are presented. The problems of modeling and evaluation based on the digital twin of the designed product are considered. The relationship between the model, on the basis of which it is supposed to evaluate the characteristics, and the models of adjacent vehicle systems, requiring a similar approach based on a digital virtual platform, is considered. Attention is paid to the main estimated parameters of the model and limitations.
\end{abstract}

\section{Introduction}

It has now become possible to use digital twins to define the characteristics of the vehicle body. Digital twins are a computer model that is built on the basis of a mathematical model that reflects the features of the object being created that are of interest to the designer or tester. Such mathematical models should be provided with the appropriate means of numerical calculations, so that they can be used to obtain adequate evaluation results.

The testing itself is performed on virtual test benches. If we supplement the computer model, which only calculates certain parameters, with additional algorithms for checking the model under various initial data and conditions, then we will get a virtual test bench. A set of virtual test benches of various functionalities can constitute a digital platform used for design, testing and various kinds of certifications [1-3].

If one equip the digital twin with additional optimization algorithms [4], additional benefits of using virtual test bench and digital platforms can be obtained. These are, for example, methods for topological optimization of the body structure [5], methods for optimizing the distribution of stiffeners, and, conversely, methods for creating lightweight structures without losing rigidity and strength.

\footnotetext{
* Corresponding author: zhilenkovanton@gmail.com
} 


\section{General concept of the digital twin of the designed product}

Defining the characteristics of the car body allows the designer to assess its rigidity and strength. These characteristics make it possible to assess the passive safety of the vehicle. This assessment is one of the most important indicators of a transport facility.

The digital twin of the vehicle body is, first of all, the definition of the general body structure, its design features, taking into account the materials used (Fig.1).

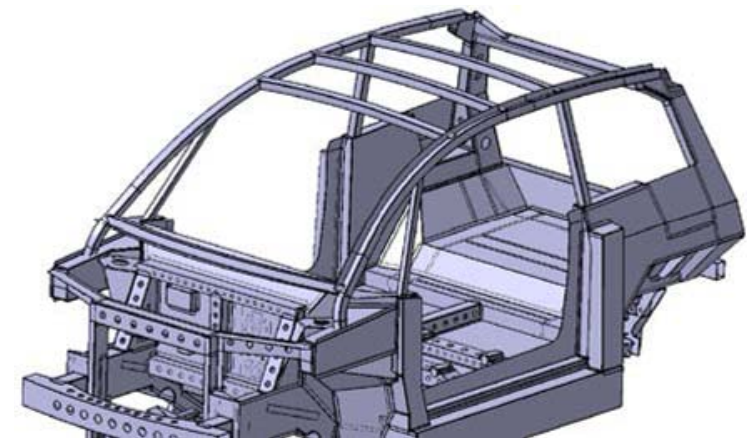

Fig. 1. General view of the car body and its structural elements.

Since the digital twin of the entire vehicle is of interest, it should also represent all other vehicle systems. First of all, these are doubles of internal systems, such as a power plant, braking system, electrical equipment, etc. (Fig. 2)

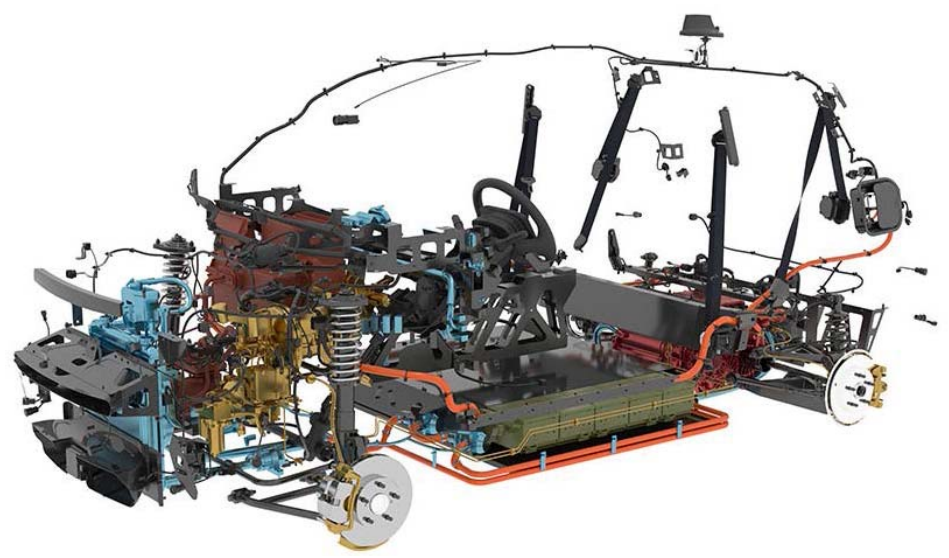

Fig. 2. The digital twin of the vehicle's internal systems.

The completeness of representation of all systems and elements of a real car in its digital twin is necessary for the possibility of implementing an integrated approach in its design and testing. This is also necessary to assess the interaction of body elements with other systems and the driver [7].

For example, in the digital twin of a car, developed at the Center of National Technological Initiative (NTI, Polytechnic University), it became possible to estimate the optimal parameters of energy storage units' location and their interaction with the car body in crash tests.

All these considerations must be taken into account when designing virtual test benches, and this leads to the need to develop many complex mathematical and computer models. These models should allow evaluating their characteristics, assessing the interaction of 
various models with each other, and allowing testing and optimization under given simulation conditions.

\section{Digital twin for assessing the parameters of the car body}

A digital twin has been developed at the Polytechnic University, which allows evaluating the mechanical rigidity and strength of the vehicle body structure (Fig. 3).

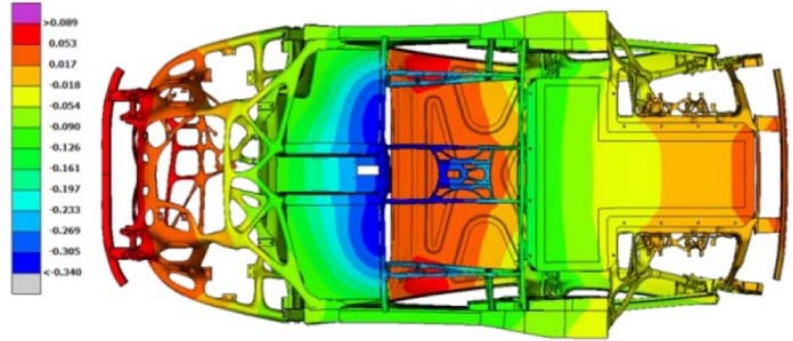

Fig. 3. The results of the analysis of the stiffness and strength of the vehicle body, obtained on a virtual bench.

The digital twin is based on a mathematical model that takes into account the mechanical characteristics of the designed body. The characteristics of the body are evaluated using this mathematical model and a 3D CAD finite element model of the car. The accuracy of the estimates obtained on the developed model is determined by the accuracy of the mathematical model itself, a sufficiently good detailing of the finite element model and computer numerical calculations [8-10].

This is an extremely complex system that allows you to significantly reduce development time, as well as significantly reduce the economic costs of conducting appropriate tests at the stage of vehicle design and certification.

The digital twin and virtual test bench should be used to assess the strength and stiffness of not only homogeneous structures, but also multi-element engineering solutions. For example, it is extremely important to be able to model the characteristics of the body, taking into account the places of attachments of its elements. It is necessary to take into account the presence of places of welding or riveting.

The developed application has a rich graphical user interface functionality. Such a complex system as a virtual test bench should provide the ability to analyze and evaluate all the critical parameters of the designed product. First of all, this requires appropriate means of visualizing the simulation results. Of course, the optimal computer system should provide the automation of some of the tasks for estimating the parameters of the designed vehicle.

Today, automating the search for the optimal topology of various mechanical systems has become an important engineering and mathematical problem. So, for example, solutions based on competitive neural networks and autoencoders allow obtaining non-traditional and non-obvious design solutions that nevertheless provide all the specified design requirements and, at the same time, surpass classical solutions in a number of parameters.

Let's consider some characteristics of the body of the designed car that can be evaluated on the virtual test benches of the digital platform. Below are some of the results obtained on the current software developed at the NTI (Polytechnic University). 


\section{Examples of obtaining characteristics on virtual test benches for cars}

The mechanical rigidity and strength of the designed product is directly related to such characteristics as the safety of the car for the driver and passengers in a collision with obstacles [11]. Safety tests of various types of frontal collisions, side collisions from the rear or the front are important here. In addition, it is necessary to carry out safety tests for impacts on the roof of the car. The latter scenario is also important for simulating rollover situations.

Figure 4 shows a virtual crash test bench for assessing the safety of the driver and passengers, which simulates a frontal impact on the left front of the car. Here the designer can assess the adequacy of the body's strength. An assessment is made of the degree of body deformation and the threat of injury to the driver or front passenger.

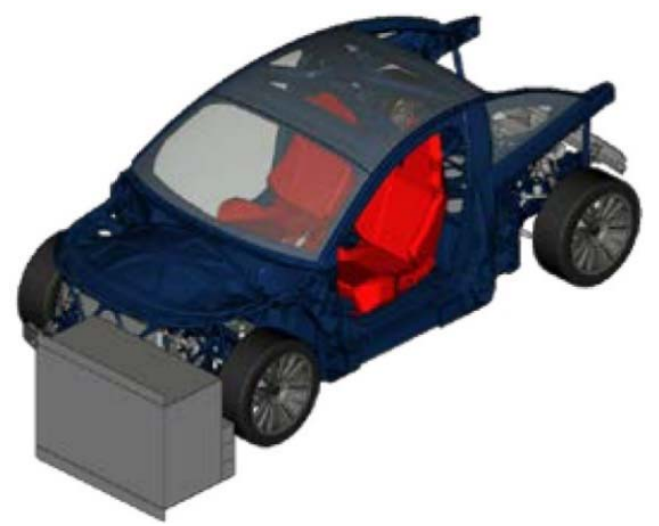

Fig. 4. An example of visualization of a crash test on a virtual test bench.

Equally important and necessary is the ability to assess the consequences of a side impact. [12] The visualization of the crash test obtained on the virtual test bench is shown in Figure 5.

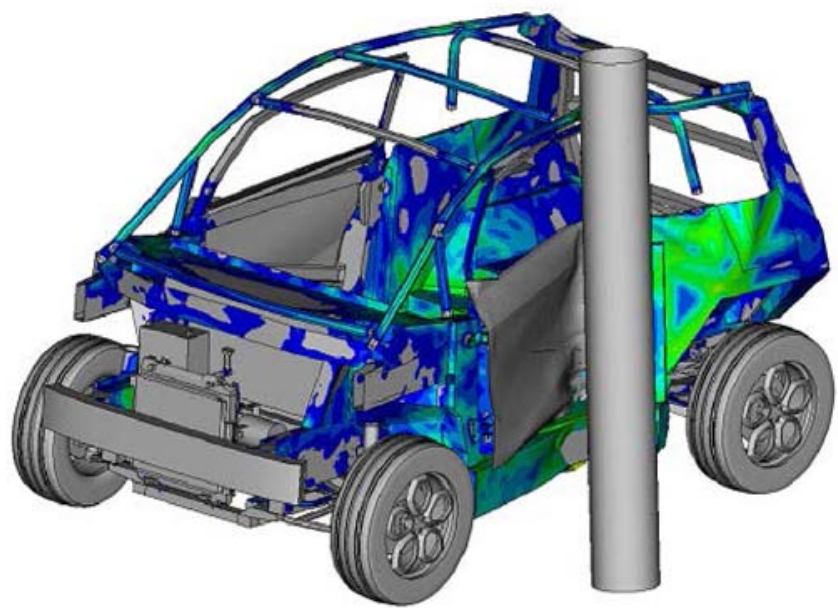

Fig. 5. Visualization of crash test simulation results where side impact is assessed.

Figure 5 simulates a side impact event for a projected electric vehicle. In this virtual test bed, the designer can assess the effects of a side impact on the vehicle's batteries. As you know, modern batteries tend to self-ignite with strong impacts. Since they tend to fill the 
underbody space under the driver and passengers, such an assessment is extremely important from the point of view of assessing the degree of safety of the vehicle being designed.

A number of other important simulations can be performed with virtual test benches. This is an assessment of the contamination of the exterior of the car, an assessment of the water resistance of the car body and drainage. Estimates of the level of acoustic noise inside the car interior also depend on the design features of the car body and the interior solutions used. When assessing the noise level, it is important to take into account the effect of the suspension on the vehicle body.

To assess the noise level, virtual test benches have been developed today, in which a digital twin of an alpha camera is implemented.

\section{Conclusion}

Modern advances in the fields of mathematical modeling, finite element models, highperformance computing, etc., have made it possible to simulate extremely complex technical systems and complexes. Digital twins built on the basis of these technologies have changed many approaches to engineering calculations, as well as to design and certification.

One of the most important problems in the design of vehicles has always been the problem of assessing the characteristics of the properties of the car body. Such estimates require complex mathematical simulations. The solution of problems of mathematical modeling includes the need to ensure the convergence of solutions, their stability.

The article shows that today such systems are realizable and allow making estimates of a given accuracy. Examples of the results of computer modeling based on a digital car model are given. It is shown that modern digital industrial technologies make it possible to create complex digital platforms with a full cycle of development of technical products.

Further research can be directed to systems for automatic optimization of designed solutions. In such systems, the parameters of the created product are set in certain intervals, the cost function is set, and the design decisions are optimized to achieve the specified parameters while minimizing (or maximizing) the given cost function.

Since it is necessary to solve the problem of ensuring conflicting requirements, the problem of multi-criteria optimization of the parameters of the designed object arises. Most often this is a non-linear problem. The promising technology here is artificial intelligence and machine learning.

\section{References}

1. A.V. Ivanov, A.A. Zhilenkov, IEEE Conference of Russian Young Researchers in Electrical and Electronic Engineering (EIConRus) 890 (2018) doi: 10.1109/EIConRus.2018.8317231

2. V.N. Kozlovsky et al, IOP Conf. Ser.: Mater. Sci. Eng. 986, 012047 (2020)

3. A.A. Zhilenkov, D. Denk, IEEE Conference of Russian Young Researchers in Electrical and Electronic Engineering (EIConRus) $1104 \quad$ (2017) doi: 10.1109/EIConRus.2017.7910748

4. D.O. Solovev et al, IOP Conf. Ser.: Mater. Sci. Eng. 747, 012076 (2020)

5. A.V. Ivanov, A.A. Zhilenkov, IEEE Conference of Russian Young Researchers in Electrical and Electronic Engineering (EIConRus) 882 (2018) doi: 10.1109/EIConRus.2018.8317229 
6. A. Bhardwaj, W. Di, J. Wei, Deep Learning Essentials: Your hands-on guide to the fundamentals of deep learning and neural network modeling (Packt Publishing Limited, Birmingham, 2018)

7. Q. Gemine, D. Ernst, B. Cornélusse, Optim Eng. 18, 587-629 (2017)

8. A.A. Zhilenkov, IEEE Conference of Russian Young Researchers in Electrical and Electronic Engineering (EIConRus) 400 (2018) doi: 10.1109/EIConRus.2018.8317117

9. S. Bai, D. Brennan, D. Dusenberry, X. Tao, Z. Zhang, SAE International Paper 201001-0368 (2010)

10. A.A. Zhilenkov, D. Denk, IEEE Conference of Russian Young Researchers in Electrical and Electronic Engineering (EIConRus) $\mathbf{1 1 0 0} \quad$ (2017) doi: 10.1109/EIConRus.2017.7910747

11. A. Leigh, J. Ronald, Institution of Electrical Engineers (2004)

12. A.A. Zhilenkov, I.R. Epifantsev, IEEE Conference of Russian Young Researchers in Electrical and Electronic Engineering (EIConRus) 1032 (2018) doi: 10.1109/EIConRus.2018.8317265 